\title{
Impact of Process-Induced Uniaxial Strain on the Temperature Dependence of Carrier Mobility in Nanoscale pMOSFETs
}

\author{
William P. N. Chen, Jack J. Y. Kuo, and Pin Su, Member, IEEE
}

\begin{abstract}
This letter provides an experimental assessment of temperature dependence of mobility for advanced short-channel strained devices. By accurate split $C-V$ mobility extraction under various temperatures, we examine the impact of process-induced uniaxial strain on the temperature dependence of mobility and mobility enhancement in nanoscale pMOSFETs. Our study indicates that the strain sensitivity of hole mobility becomes less with increasing temperature, and it is consistent with previous mechanical-bending result. Furthermore, the carrier-scattering mechanism for the pMOSFET under uniaxial compressive strain tends to be more phonon limited at a given vertical electric field, which explains the larger drain current sensitivity to temperature present in the compressively strained PFET.
\end{abstract}

Index Terms-Mobility, MOSFET, strain silicon, temperature dependence.

\section{INTRODUCTION}

$\mathbf{U}$ NIAXIAL strained-Si technology is crucial to transistor performance in state-of-the-art CMOS development [1]-[3]. The temperature effect on strain-enhanced mobility is of special importance because it may provide insights for the underlying mechanisms responsible for the performance enhancement. Several studies have investigated the temperature effect on strain-enhanced mobility in the past [4]-[7]. For NMOS, the temperature effect of process-induced biaxial strain [4], [5], uniaxial strain [4], and mechanical uniaxial strain [6] applied on devices has been experimentally studied. The results all indicated less strain sensitivity in carrier mobility with decreasing temperature. For PMOS, the temperature effect of process-induced biaxial strain [5] and mechanical uniaxial strain [7] has also been investigated. The results showed higher strain sensitivity in carrier mobility with decreasing temperature, i.e., an opposite trend with NMOS. However, the temperature effect of process-induced uniaxial strain in nanoscale PMOS devices is still not reported and merits investigation.

Manuscript received September 17, 2009; revised January 5, 2010 and February 11, 2010. Date of publication April 5, 2010; date of current version April 23, 2010. This work was supported in part by the National Science Council of Taiwan under Contract NSC98-2221-E-009-178 and in part by the Ministry of Education in Taiwan under the ATU Program. The review of this letter was arranged by Editor L. Selmi.

The authors are with the Department of Electronics Engineering and Institute of Electronics, National Chiao Tung University, Hsinchu 30013, Taiwan (e-mail: williamchen.ee93g@nctu.edu.tw; jack.ee93g@nctu.edu.tw; pinsu@faculty.nctu.edu.tw).

Color versions of one or more of the figures in this letter are available online at http://ieeexplore.iee.org.

Digital Object Identifier 10.1109/LED.2010.2044553
In this letter, we conduct an experimental assessment for the impact of the process-induced uniaxial strain on the temperature dependence of carrier mobility in nanoscale pMOSFETs.

\section{EXPERIMENTAL SETUP}

pMOSFETs with channel direction $\langle 110\rangle$ with neutral, tensile, and compressive uniaxial contact Etch-Stop Layer (CESL) were manufactured based on the state-of-the-art CMOS technology on a 300-mm (100) silicon substrate [8], [9]. Shallow Trench Isolation (STI) was patterned to define the active region. A uniform channel doping was implanted to define the PMOS devices and maintain reasonable threshold voltage. Then an ultrathin nitride oxide was grown on the surface of the wafer. The polygate was implanted with heavily doped $\mathrm{P}+$ species and postannealed to increase the gate activation rates. Then the ultrashallow Highly Doped Drain (HDD) implant, oxide/nitride spacer formation, source and drain implant, postimplantation annealing, and back-end process were implemented sequentially. The effective channel doping is around $2 \times 10^{18} \mathrm{~cm}^{-3}$ for short-channel devices based on TCAD simulation. The equivalent oxide thickness is about $17 \AA$. For comparison purposes, nitride films with neutral, -2.8-GPa compressive strain, and 1.6-GPa tensile stress were deposited on pMOSFETs at the CESL deposition stage. The details about our TCAD simulation are in [15].

Since the external resistance $\left(R_{\mathrm{Sd}}\right)$ is crucial to the shortchannel mobility extraction [10], [11], the intrinsic drain current $\left(I_{D}\right)$ was calibrated by considering the series-resistance effect [8]. The physical polygate length $\left(L_{\text {phy }}\right)$ was obtained using inline SEM measurement. The LDD overlap region under the gate $\left(L_{\mathrm{ov}}\right)$ was extracted by the split $C-V$ method [14]. The effective channel length $\left(L_{\mathrm{EFF}}\right)$ can then be derived by subtracting $L_{\mathrm{ov}}$ from $L_{\mathrm{phy}}$. Finally, the mobility for shortchannel devices can be determined [8]. In this letter, PMOS devices with effective channel length $L_{\mathrm{EFF}}=95 \mathrm{~nm}$ were measured with temperature ranging from 100 to $300 \mathrm{~K}$.

\section{Results AND Discussion}

Fig. 1 shows the drain current $\left(I_{D}\right)$ versus gate voltage characteristics at various temperatures for the PMOS devices under test. The drain current shows strong correlation with stressor types and can be explained by the extracted carrier mobility, as shown in Fig. 2. It can be seen from Fig. 2 that the shortchannel mobility in PMOS $\left(L_{\mathrm{EFF}}=95 \mathrm{~nm}\right)$ shows significant dependence on the uniaxial strain. The PMOS mobility prefers 


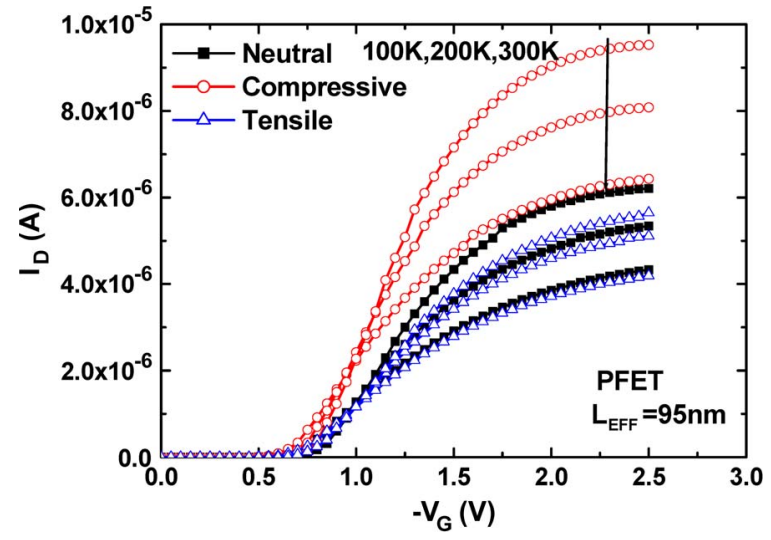

Fig. 1. Drain current versus gate voltage at various temperatures for PFETs with various stressors. The drain bias $\left(V_{\mathrm{DS}}\right)$ is $-5 \mathrm{mV}$. The temperature dependence of the drain current shows strong correlation with stressor types. The length of diffusion is $0.29 \mu \mathrm{m}$.

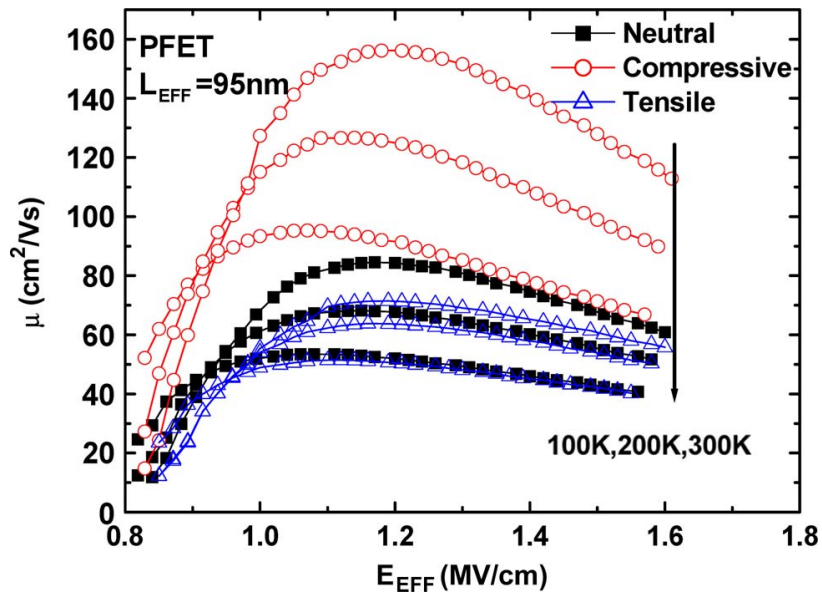

Fig. 2. Extracted carrier mobility shows significant dependence on the uniaxial stressor.

compressive stress because of the strain-reduced conductivity effective mass [9].

In addition, Fig. 2 shows that the mobility is degraded when temperature increases in the high vertical-field region, where phonon scattering is important [12], [13]. Moreover, the temperature dependence of mobility shows strong sensitivity to strain. In other words, as the mobility is enhanced by compressive strain, its temperature dependence also increases.

Fig. 3 shows the temperature sensitivity $(\log \mu / \log T)$ of the hole mobility versus the vertical effective electric field $\left(E_{\mathrm{EFF}}\right)$. For a given stressor, it can be seen that the temperature sensitivity increases (i.e., more negative) and then saturates as $E_{\mathrm{EFF}}$ increases. More importantly, the $\log \mu / \log T$ for the PFET under compressive strain is the highest in absolute value among the three stressors. In other words, the scattering mechanism of the PMOS device becomes more phonon limited [12], [13] under compressive strain. This also explains why the temperature sensitivity of drain current for the compressively strained PFET is the largest among the three stressors, as shown in Fig. 1.

Fig. 4 shows the hole-mobility enhancement $(\Delta \mu / \mu)$ versus temperature at $E_{\mathrm{EFF}}=1.5 \mathrm{MV} / \mathrm{cm}$. It shows that for both compressive and tensile stressors, the magnitude of $\Delta \mu / \mu$ decreases as temperature increases. Our result from the process-induced

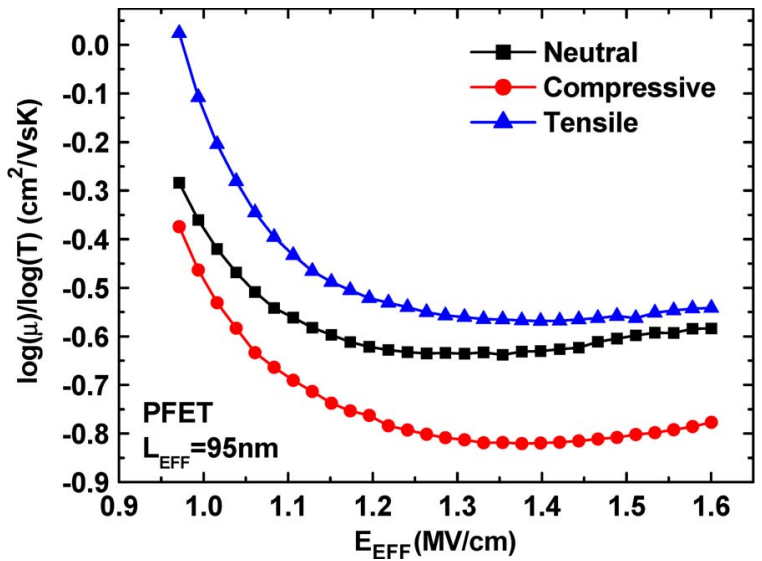

Fig. 3. Temperature sensitivity of hole mobility versus the vertical field for various uniaxial stressors.

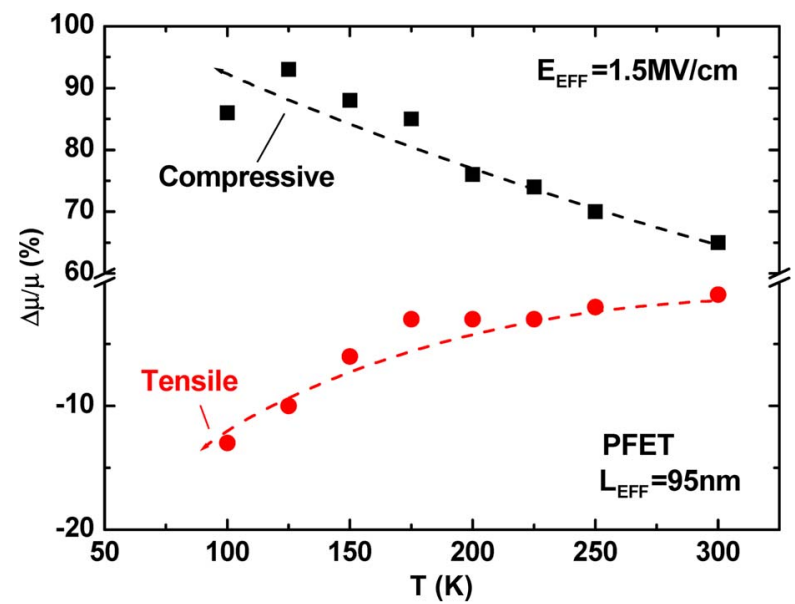

Fig. 4. Hole-mobility enhancement versus temperature at $E_{\mathrm{EFF}}=$ $1.5 \mathrm{MV} / \mathrm{cm}$ for PMOS devices with various stressors.

uniaxial stressors is consistent with the study in [7], in which an external compressive uniaxial mechanical stress was applied.

Based on the model proposed in [7], it is plausible that as temperature increases, the compressively strained PFET has less holes to populate states near the band edge where the conductivity effective mass along the channel direction is smaller. Therefore, the observed mobility enhancement decreases with increasing temperature.

\section{CONCLUSION}

We have investigated the temperature dependence of mobility for advanced short-channel strained PMOS devices. By accurate split $C-V$ mobility extraction under various temperatures, we examine the impact of process-induced uniaxial strain on the temperature dependence of mobility and mobility enhancement in nanoscale pMOSFETs. Our study indicates that the strain sensitivity of hole mobility becomes less with increasing temperature, and it is consistent with previous uniaxial mechanical-bending result. Furthermore, the carrier-scattering mechanism for the pMOSFET under uniaxial compressive strain tends to be more phonon limited at a given vertical electric field, which explains the larger drain current sensitivity to temperature present in the compressively strained PFET. 


\section{REFERENCES}

[1] Z. Luo, N. Rovedo, S. Ong, B. Phoong, M. Eller, H. Utomo, C. Ryou, H. Wang, R. Stierstorfer, L. Clevenger, S. Kim, J. Toomey, D. Sciacca, J. Li, W. Wille, L. Zhao, L. Teo, T. Dyer, S. Fang, J. Yan, O. Kwon, D. Park, J. Holt, J. Han, V. Chan, J. Yuan, T. Kebede, H. Lee, S. Kim, S. Lee, A. Vayshenker, Z. Yang, C. Tian, H. Ng, H. Shang, M. Hierlemann, J. Ku, J. Sudijono, and M. Ieong, "High performance transistors featured in an aggressively scaled 45-nm bulk CMOS technology," in VLSI Symp. Tech. Dig., 2007, pp. 16-17.

[2] F. Andrieu, T. Ernst, F. Lime, F. Rochette, K. Romanjek, S. Barraud, C. Ravit, F. Boeuf, M. Jurczak, M. Casse, O. Weber, L. Brevard, G. Reimbold, G. Ghibaudo, and S. Delenibus, "Experimental and comparative investigation of low and high field transport in substrate- and process-induced strained nanoscaled MOSFETs," in VLSI Symp. Tech. Dig., 2005, pp. 176-177.

[3] E. X. Wang, P. Matagne, L. Shifren, B. Obradovic, R. Kotlyar, S. Cea M. Stettler, and M. D. Giles, "Physics of hole transport in strained silicon MOSFET inversion layers," IEEE Trans. Electron Devices, vol. 53, no. 8, pp. 1840-1851, Aug. 2006.

[4] F. Lime, F. Andrieu, J. Derix, G. Ghibaudo, F. Boeuf, and T. Skotnicki, "Low temperature characterization of effective mobility in uniaxially and biaxially strained nMOSFETs," Solid State Electron., vol. 50, no. 4, pp. 644-649, Apr. 2006

[5] N. Sugii and K. Washio, "Low-temperature electrical characteristics of strained-Si MOSFETs," Jpn. J. Appl. Phys., vol. 42, pt. 1, no. 4B, pp. 1924-1927, 2003.

[6] M. N. Tsai, T. C. Chang, P. T. Liu, O. Cheng, and C. T. Huang, "Temperature effects of n-MOSFET devices with uniaxial mechanical strains," Electrochem. Solid-State Lett., vol. 9, no. 8, pp. G276-G278, Jun. 2006.

[7] X. Yang, S. Parthasarathy, Y. Sun, A. Koehler, T. Nishida, and S. E. Thompson, "Temperature dependence of enhanced hole mobility in uniaxial strained p-channel metal-oxide-semiconductor field-effect transistors and insight into the physical mechanisms," Appl. Phys. Lett., vol. 93 , no. 24 , p. 243503 , Dec. 2008.

[8] W. P. N. Chen, P. Su, and K. Goto, "Impact of process-induced strain on Coulomb scattering mobility in short-channel n-MOSFETs," IEEE Electron Device Lett., vol. 29, no. 7, pp. 768-770, Jul. 2008.

[9] S. E. Thompson, G. Sun, Y. S. Choi, and T. Nishida, "Uniaxial-processinduced strained-Si: Extending the CMOS roadmap," IEEE Trans. Electron Devices, vol. 53, no. 5, pp. 1010-1020, May 2006.

[10] S. Severi, L. Pantisano, E. Augendre, E. San Andrés, P. Eyben, and K. De Meyer, "A reliable metric for mobility extraction of short-channel MOSFETs," IEEE Trans. Electron Devices, vol. 54, no. 10, pp. 26902698, Oct. 2007.

[11] D. Fleury, A. Cros, G. Bidal, J. Rosa, and G. Ghibaudo, "A new technique to extract the source/drain series resistance of MOSFETs," IEEE Electron Device Lett., vol. 30, no. 9, pp. 975-977, Sep. 2009.

[12] K. Chain, J.-H. Huang, J. Duster, P.K. Ko, and C. Hu, "A MOSFET electron mobility model of wide temperature range $(77-400 \mathrm{~K})$ for IC simulation," Semicond. Sci. Technol., vol. 12, no. 4, pp. 355-358, Apr. 1997.

[13] S.-I. Takagi, A. Toriumi, M. Iwasw, and H. Tango, "On the universality of inversion layer mobility in Si MOSFETs: Part II-Effects of surface orientation," IEEE Trans. Electron Devices, vol. 41, no. 12, pp. 23632368, Dec. 1994.

[14] A. Cros, K. Romanjek, D. Fleury, S. Harrison, R. Cerutti, P. Coronel, B. Dumont, A. Pouydebasque, R. Wacquez, B. Duriez, R. Gwoziecki, F. Boeuf, H. Brut, G. Ghibaudo, and T. Skotnicki, "Unexpected mobility degradation for very short devices: A new challenge for CMOS scaling," in IEDM Tech. Dig., 2006, pp. 1-4.

[15] W. P.-N. Chen, P. Su, and K.-I. Goto, "Investigation of Coulomb mobility in nanoscale strained PMOSFETs," IEEE Trans. Nanotechnol., vol. 7, no. 5, pp. 538-543, Sep. 2008. 